\title{
Multiple-Model Adaptive Control - Disturbance Rejection Study
}

\author{
Ya Wang ${ }^{1, a}$, Zhuo Kong ${ }^{2, b}$ and Baoyong Zhao ${ }^{3, c}$ \\ ${ }^{1}$ University of Science \& Technology Beijing, China \\ ${ }^{2}$ University of Science \& Technology Beijing, China \\ ${ }^{3}$ University of Science \& Technology Beijing, China \\ awangwangya@yeah.net, bjustwe137@hotmail.com, 'czhby@ustb.edu.cn
}

Keywords: MMAC; weighting algorithm; multiple-model adaptive control.

Abstract. This paper is concerned with the disturbance rejection of weighted multiple-model adaptive control (WMMAC), in which the error index of weighting algorithm has been improved to filter out the influence of severe disturbance. Simulation results verified the effectiveness of the proposed scheme.

\section{Introduction}

Multiple model concepts in adaptive estimation and control originated from D. T. Magill [1]. Up to now, multiple model adaptive control (MMAC), especially WMMAC is an effective approach of robust adaptive control. There are many research results since its origination around 1960s, see [2-21] for example among others. In structure, WMMAC has much similarities with T-S model based fuzzy control (the parallel distributed compensation), and possesses some advantages against T-S model based fuzzy control, since WMMAC uses on-line information to calculate weights signals, the T-S model based fuzzy control (the parallel distributed compensation) uses off-line information (membership function) to calculate weights signals. Fig. 1 describes the basic logic structure of a WMMAC system.

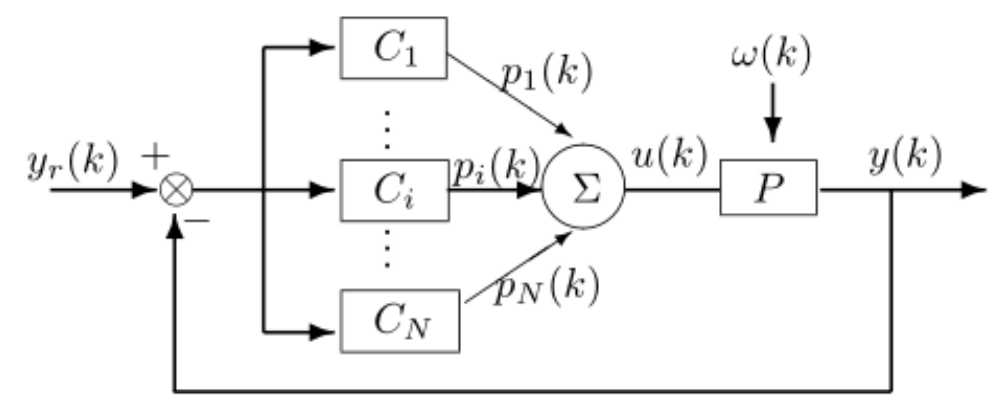

Fig.1. Simplified block diagram of a general WMMAC system

In Fig. $1, C_{i}$ is a local controller; $p_{i}(k)$ is the weighting coefficient to the $i^{\text {th }}$ plant model $M_{i}$ and then used as the controller weight of $C_{i}$. Denote $C_{i} \in C . C=\left\{C_{i}, i=1,2, \ldots N\right\}$ is the controller set. And $C_{i}$ is designed according to model $M_{i} \in M M=\left\{M_{i}, i=1,2, \ldots N\right\}$ is the model set that covers the uncertainty of plant $p$. The control objective is to track the bounded reference input $y_{r}(k)<\infty$.

It is well known that a weighting algorithm plays an important role in WMMAC systems. As the author of reference [17] pointed out, the closed-loop stability of a WMMAC system mainly depends on three conditions: 1 ) the 'local' model set includes the true model of the plant or the closet one to the plant; 2) the weighting algorithm converges correctly to identify the closet 'local' model and the corresponding 'local' controller; 3) each 'local' controller stabilizes its corresponding 'local' model. In most simulation cases, the weighting algorithm proposed in [17] has satisfactory robustness against noises with appropriate signal to noise ratio. But it is also observed that when the signal to noise ratio exceeds a certain lower level the weights signals do not converge correctly. 
Motivated by this observation, this paper is intended to further improve the convergence performance of a weighting algorithm against high level disturbance (noise).

The reminder of the paper is organized as follows. Section 2 gives the description of a general multiple-model adaptive control system for a discrete-time stochastic plant. Section 3 presents the weighting algorithm with focus on the improvement of error index to leave out the influence of disturbance or noise. Section 4 is the main results of the stability and convergence of the resulting closed-loop MMAC system. Section 5 includes some simulation results to verify the effectiveness of the proposed method. And finally, the conclusions are given in Section 6.

To avoid ambiguity, we emphasize that the stability of the weighted MMAC system means the bound of its input-output signals; and the convergence of the weighted MMAC system means that the input-output signals of the MMAC system converge to that of the non-adaptive system consisted of one of the 'local' controllers and the plant.

In addition, we need to point out that all the limit operations in this paper are in the sense of probability one.

\section{Description of the multiple-model adaptive control}

Consider the following time-varying or uncertain discrete-time stochastic plant $P$ with single input and single output (SISO)

Where

$$
A\left(k, q^{-1}\right) y(k)=q^{-d} B\left(k, q^{-1}\right) \mu(k)+\omega(k) .
$$

$$
A\left(k, q^{-1}\right)=1+a_{1}(k) q^{-1}+\cdots a_{n}(k) q^{-n} \quad B\left(k, q^{-1}\right)=b_{0}(k)+b_{1}(k) q^{-1}+\cdots b_{m}(k) q^{-m}
$$

Where $y(k), \mu(k), \omega(k)$ are the output, input and zero-mean white noise of the system, respectively, $y(k)=0, \mu(k)=0, \omega(k)=0$ for $k<0$, and that

$$
\lim _{n \rightarrow \infty} \frac{1}{n} \sum_{i=1}^{n}[\omega(i)]^{2}=R<\infty .
$$

The plant $P$ can be stable or non-stable, minimum phase or non-minimum phase. Its output $y(k)$ can be rewritten as

$$
y(k)=\phi^{T}(k-d) \theta(k)+\omega(k) .
$$

Where

$$
\begin{gathered}
\phi^{T}(k-d)=[y(k-1), \ldots, y(k-n), u(k-d), \ldots, u(k-d-m)] . \\
\theta(k)=\left[-a_{1}(k), \ldots,-a_{n}(k),-b_{0}(k),-b_{1}(k), \ldots,-b_{m}(k)\right] .
\end{gathered}
$$

The time-varying character or uncertainty of the plant is limited to the following situation. $\theta(k)$ is assumed piecewise continuous, uniformly bounded in time, thus a finite set of candidate parameter values $\Theta:=\vartheta_{1}, \vartheta_{2}, \ldots, \vartheta_{N}$ indexed by $i \in 1, \ldots, N$ can cover the uncertainty of the plant. Each $\vartheta_{i}$ corresponds to a 'local' model $M_{i}$.

For each model $M_{i} \in M$, its output is given by

$$
y_{i}(k)=\phi^{T}(k-d) \theta_{i} .
$$

Further, define the output error of each model $M_{i}$ i.e.

$$
e_{i}(k)=y(k)-y_{i}(k)=y(k)-\phi^{T}(k-d) \theta_{i} .
$$

As stated before, the key point of MMAC is to construct an effective weighting algorithm which will be described in the next section.In the previous work [17], $e_{i}(k)$ is the only error index, based on which the weighting algorithm recursively generates the weights signal $p_{i}(k)$.

In this paper, an alternative error index will be adopted when the system output $y_{k}(k)$ enters the scope of steady state, for details, see the next section. As to 'local' controller $C_{i}$, it is designed according to $M_{i}$ based on pole placement strategy, for details, see the SIMULATION section. 


\section{Weighting algorithm}

Weighting algorithms play important roles in WMMAC, as well as multiple model adaptive estimation (WMMAE) .The classical weighting algorithm, which is based on multiple Kalman filters, dynamic hypothesis testing, and Bayesian formula, has been replace by a novel weighting algorithm proposed in [17]. In the sense of complexity and computation burden, the new algorithm possesses some advantages against the classical algorithm, and is much easier to analyze and implement, besides the benefits of leaving out the Kalman filters.

The weighting algorithm put forward in [17] can be described as follows.

$$
\begin{gathered}
l_{i}(0)=\frac{1}{N} ; p_{i}(0)=l_{i}(0) . \\
l_{i}^{\prime}(k)=1+\frac{1}{k} \sum_{p=1}^{k} e_{i}(p)^{2} . \\
l_{\min }(k)=\min _{i} l_{i}^{\prime}(k) . \\
l_{i}(k)=l_{i}(k-1) \frac{l_{\min }(k)}{l_{i}^{\prime}(k)} . \\
p_{i}(k)=\frac{l_{i}(k)}{\sum_{i=1}^{N} l_{i}(k)}
\end{gathered}
$$

According to [17], we have the convergence result.

Theorem $1^{[17]}$ : If the model closest to the true plant in the following sense with probability one

$$
\left\{\begin{array}{c}
\sum_{r=1}^{k} e_{j}^{2}(r)<\sum_{r=1}^{k} e_{i}^{2}(r), \forall k \geq d+1 \\
\frac{1}{k} \sum_{r=1}^{k} e_{j}^{2}(r) \rightarrow S_{j} \\
\frac{1}{k} \sum_{r=1}^{k} e_{i}^{2}(r) \rightarrow S_{i} \\
S_{j}<S_{i}, i \neq j
\end{array} .\right.
$$

where $S_{j}$ is a constant, $S_{i}$ may be a constant or infinity.

Then the weighting algorithm (3.1)-(3.5) leads to

$$
p_{j}(k) \rightarrow 1 ; p_{i}(k) \rightarrow 0, i=1, \ldots N, i \neq j \text {. }
$$

To adapt the above-mentioned weighting algorithm to high level noise, we need to redefine the error signal as

$$
\left\{\begin{array}{c}
e_{i}(k)=y_{r}(k)-y_{i}(k),\left|\frac{y_{r}(k)-y(k)}{y_{r}(k)}\right|<\gamma, y_{r}(k) \neq 0 \\
e_{i}(k)=y(k)-y_{i}(k), \text { otherwise }
\end{array} .\right.
$$

Generally speaking, the value of $\gamma$ depends on the level of noise relatively to the reference signal, i.e, the signal-to-noise ratio, and the choice of error band ( $\pm 5 \%$ or $\pm 3 \%$ ).In real engineering, it can be determined by trial and error.

Remark: the reason for the new error index to leave out the influence of noise can be observed from (15). In $y_{r}(k)$, and $y_{i}(k)$ there are no noise influence, but $y(k)$ is obviously influenced by noise $\omega(k)$. So, by using $e_{i}(k)=y_{r}(k)-y_{i}(k)$ after the system entering the scope of steady state, the weighting algorithm could get rid of system disturbance or noise. On the other hand, it is also worth pointing out that to thoroughly replace $e_{i}(k)=y_{r}(k)-y_{i}(k)$ with $e_{i}(k)=y_{r}(k)-y_{i}(k)$ is not feasible. That is because, taking step response as an example, at the beginning stage of system dynamic 
process, each $e_{i}(k)=y_{r}(k)-y_{i}(k)$ is almost the same. And the differences among 'local' models can't be suitably reflected by $e_{i}(k)=y_{r}(k)-y_{i}(k)$.

\section{Simulation result}

To test the effectiveness of the new error index and the weighting algorithm, and the performance of the resulting closed-loop WMMAC system, two cases of simulations have been conducted with MATLAB version 6.5 .

Consider the following uncertain discrete-time stochastic plant

$$
\left(1+a_{1} q^{-1}+a_{2} q^{-2}\right) y(k)=q^{-1}\left(b_{0}+b_{1} q^{-1}\right) \mu(k)+\omega(k) .
$$

where $\omega(k)$ is a zero-mean white noise sequence with variance $\sigma=10$ that was generated by the randn() function. The deterministic part of (18) is obtained by converting the following continuous-time LTI model to a discrete-time model with sampling time $t_{2}=0.5 \mathrm{~s}$ and the zero order hold.

$$
\frac{K}{s^{2}-3 s+2} \text {. }
$$

The uncertainty of (16) comes from $K$ in (17). For the real plant model, we have $K=1.255$.The model set includes four models, corresponding to $K=K_{1}, K=K_{2}, K=K_{3}, K=K_{4}$, respectively. Four 'local' controllers were designed according to pole assignment strategy. Each controller stabilizes its corresponding model by formulating an expected closed-loop characteristic polynomial $A_{m}\left(q^{-1}\right)$ and track the reference signal $y_{r}(k)$.

$$
A_{m}\left(q^{-1}\right)=1-1.3205 q^{-1}+0.4966 q^{-2} .
$$

which corresponds to the characteristic polynomial of the following continuous time second-order system

$$
\frac{w_{n}^{2}}{s^{2}+2 \varepsilon w_{n}+w_{n}^{2}} \text {. }
$$

with $\varepsilon=0.707, w_{n}=1$, and sampling time $t_{s}=0.5$.

In addition, we adopt $\gamma=0.1$ in error index (15).

Case 1: $K_{1}=0.7, K_{2}=0.8, K_{3}=1.2, K_{4}=1.3$. Error index $e_{i}(k)=y(k)-y_{i}(k)$ is adopted. The simulation results, i.e., the four weights signals, the closed-loop output $y(k)$ against reference signal $y_{r}(k)$, and the control signal $u(k)$ are shown in Fig. 2 and Fig. 3, respectively.

Case 2: $K_{1}=0.7, K_{2}=0.8 K_{3}=1.2, K_{4}=1.3$. Error index (15) is adopted. The simulation results are shown in Fig. 4 and Fig. 5, respectively.

The closest model is the $4^{\text {th }}$ model with $K_{4}=1.3$, since the real model is with $K=1.255$. In Case 1 , the four controller weights signals $p_{i}(k), i=1,2,3,4$ can't converge correctly. In Case 2 , the four controller weights signals $p_{i}(k), i=1,2,3,4$ can still converge correctly.

\section{Conclusion}

To enhance the robustness of the weighting algorithm against disturbance/noise in WMMAC systems, a new error index is proposed. Both theoretical analysis and simulation results verified the effectiveness of the proposed scheme. The future work will be focused on improving further the error index and the weighting algorithm to get more robust performance against different kinds of disturbances and noises, and to remain rapid convergence rate at the same time. 

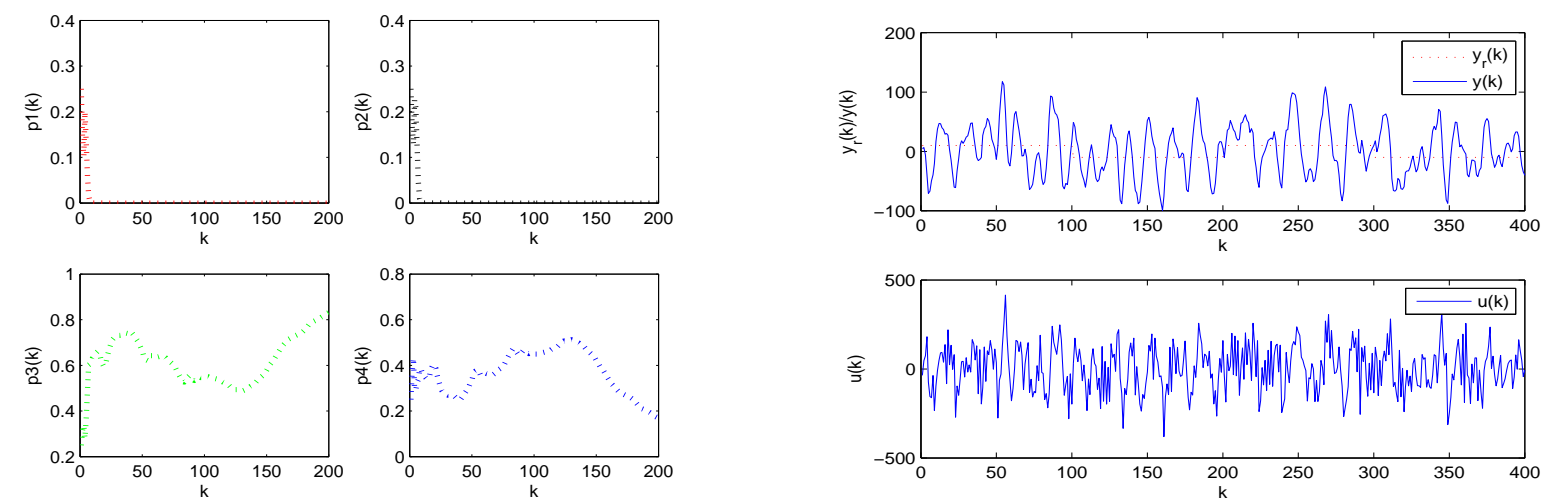

Figure. 2. The weights signals $p_{i}(k)$ in case 1 Figure. 3 . The output and control signals in case 1
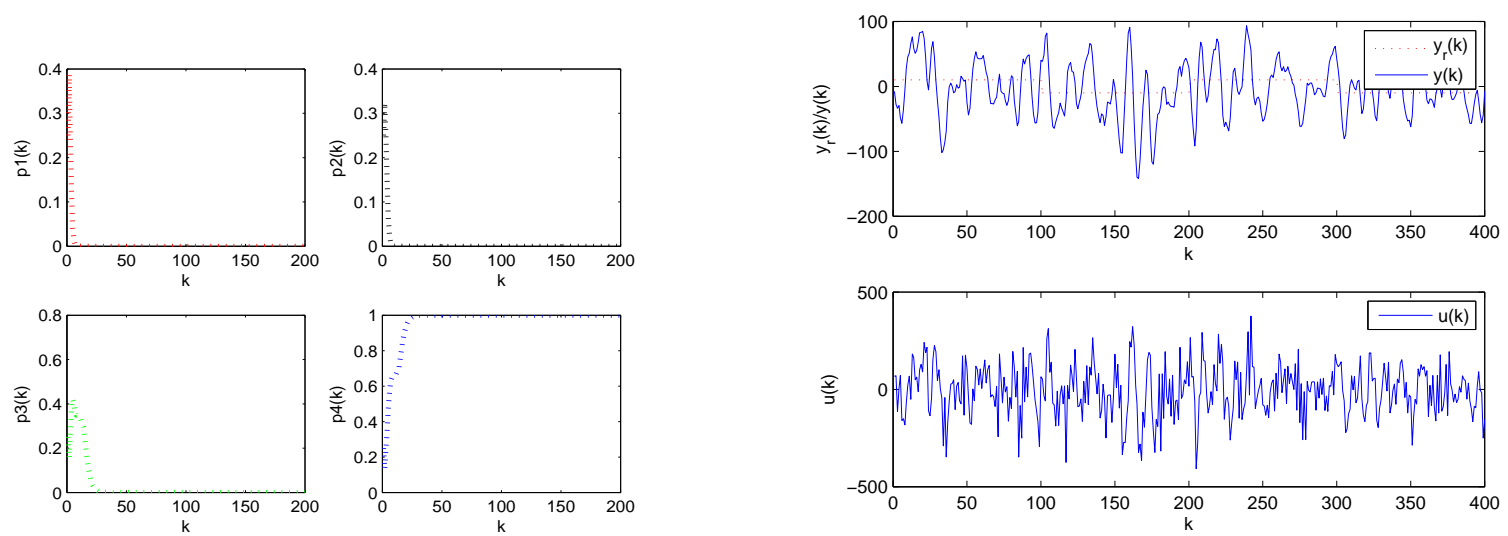

Figure. 4. The weights signals $p_{i}(k)$ in case 2

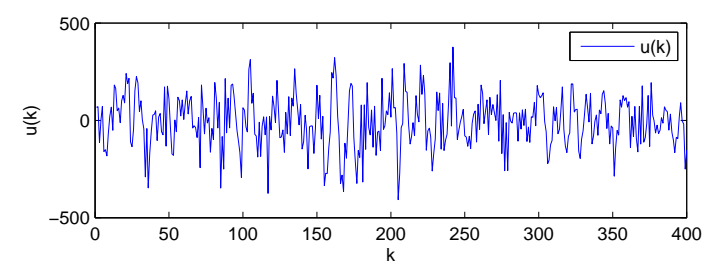

Figure. 5. The output and control signals in case 2

\section{References}

[1] D. T. Magill. Optimal adaptive estimation of sampled stochastic processes. IEEE Trans. on Automat. Contr., 10:434-439 (1965).

[2]D. G. Lainiotis. Partitioning: A unifying framework for adaptive systems I: Estimation II: Control. IEEE Trans. on Automat. Contr., 64:1182-1198 (1976).

[3] M.Athans, et. al. The stochastic control of the F-8c air-craftusing amultiplemodel adaptive control(mmac) method part I: Equilibrium flight. IEEE Trans. on Automat. Contr.,22:768- 780 (1977).

[4] M. Athans,et. al. Investigation of the multiple method adaptive control (MMAC) method for flight control systems. Technical Report NASA-CR-2916, NASA Technical Reports (1979)

[5] B. D. O. Anderson and J. B. Moore. Optimal Filtering. Prentice Hall, New Jersey, USA (1979).

[6] X. R. Li and Y. Bar-Shalom. Multiple-model estimation with variable structure. IEEE Trans. on Automat. Contr., 41:478-493 (1996).

[7] B. D. O. Anderson, et. al. Multiple model adaptive control: PartI: Finite controller coverings. Int. J. of Robust and Nonlinear Control,10:909-929 (2000).

[8] M. Athans, S. Fekri, and A. Pascoal. Issues on robust adaptive feedback control. In Preprints 16th IFAC World Congress, Invited Plenary paper, 9-39, Prague, Czech Republic (2005).

[9] S. Fekri, M. Athans and A. Pascoal, "Issues, progress and new results in robust adaptive control," Int. J. Adapt. Control and Signal Process, Vol. 20(10), pp.519-579 (2006).

[10] S. Fekri, M. Athans and A. Pascoal, "Robust multiple model adaptive control (RMMAC): 
A case study," Int. J. Adapt. Control and Signal Process, Vol. 21(1), pp.1-30 (2007).

[11] J. Hespanha, D. Liberzon, A. S. Morse, et. al. Multiple model adaptive control: Part II: Switching. Int. J. of Robust and Nonlinear Control, 11: 479-496 (2001).

[12] A.S. Morse. Supervisory control of families of linear set-point controllers - Part I: Exact matching. IEEE Trans. on Automat. Contr., 41:1413-1431 (1996).

[13] A. S. Morse. Supervisory control of families of linear set-point controllers - Part II: Robustness. IEEE Trans. on Automat. Contr., 42:1500-1515 (1997).

[14] K. S. Narendra and J. Balakrishnan. Adaptive control using multiple models. IEEE Trans. on Automat. Contr., 42:171-187 (1997).

[15] K. S. Narendra and O. A. Driollet. Stochastic adaptive control using multiple models for improved performance in the presence of random disturbances. Int. J. Adapt. Control Signal Process., 15:287-317 (2001).

[16] G. J. Schiller and P. S. Maybeck. Control of a large space structure using MMAE/MMAC techniques. IEEE Transactions on Aerospace and Electronic System, 33:1122-1131 (1997).

[17] Weicun Zhang, Stable Weighted Multiple Model Adaptive Control: Discrete-Time Stochastic Plant, International Journal of Adaptive Control and Signal Processing, 27(7): 562-581 (2013). 